Ext er nal doubl e ref er ence net hod to st udy concent $r$ at $i$ on and temper at ure dependences of chem cal shifts determined on a uni fied scal e

\begin{tabular}{|l|l|}
\hline 著者 & M zuno Kazuko, Tam ya Yuka, Nekat a Namor u \\
\hline $\begin{array}{l}\text { j our nal or } \\
\text { publ i cat i on ti t l e }\end{array}$ & Pur e and Appl i ed Chemi stry \\
\hline vol une & 76 \\
\hline number & 1 \\
\hline page range & $105-114$ \\
\hline year & 2004 01 \\
\hline URL & ht t p: //hdl . handl e. net /10098/1046 \\
\hline
\end{tabular}




\title{
External double reference method to study concentration and temperature dependences of chemical shifts determined on a unified scale*
}

\author{
Kazuko Mizuno ${ }^{1, \ddagger}, Y_{\text {Yka Tamiya }}^{1}$, and Mamoru Mekata ${ }^{2}$ \\ ${ }^{1}$ Department of Applied Chemistry and Biotechnology, Faculty of Engineering, \\ University of Fukui, Fukui 910-8507, Japan; 22-13-5 Kitaoji, Otsu 520-0843, Japan
}

\begin{abstract}
We present the principle of an external reference method of NMR, named the external double reference method, to study concentration and temperature dependences of chemical shifts. By the method, we refer chemical shifts of various samples at different temperatures to the peak of a reference substance at a fixed referring temperature. Thus, we can determine the chemical shifts in a variety of sample conditions on a unified scale. Results of the application of the method to ${ }^{1} \mathrm{H}$ - and ${ }^{13} \mathrm{C}$-chemical shifts of water and methanol are presented. We also report new data of volume magnetic susceptibilities for several deuterated solvents useful for the calibration in the method.
\end{abstract}

\section{INTRODUCTION}

Chemical shifts of ${ }^{1} \mathrm{H}$ - and ${ }^{13} \mathrm{C}$-NMR have provided rich information about microscopic states of organic compounds in various environments [1]. Especially, it is quite important to measure chemical shifts for the study of weak intermolecular interaction as hydrogen bonds. Since the applied magnetic field at the sample for NMR measurements cannot be always precisely determined, it is common to evaluate the chemical shift on the basis of an NMR peak of a well-known reference substance.

The reference substance may be mixed with the sample solution. This method called the internal reference method has an inevitable problem in the case of a liquid sample; even rather inert reference substances, such as tetramethylsilane (TMS), interact with the sample so that the reference signal may be affected by solvation. This gives a fatal difficulty in studying the concentration or temperature dependence of the chemical shift by this method.

The external reference method using a tube filled with a reference substance is an alternative method and getting more popular because one need not worry about the interaction between the sample solution and the reference substance. Still, we have a serious problem characteristic of the technique which utilizes local magnetic field at a nuclear spin in the sample. Each nuclear spin is subject to a Lorentz field and a demagnetizing field due to the magnetic polarization produced by the intense magnetic field of the superconducting magnet, in addition to the hyperfine field produced by the electrons in the molecule, which brings about the chemical shift and is our central interest. Accordingly, to measure the chemical shift, it is necessary to correct the observed shift for the contributions from the Lorentz field and the demagnetizing field. Since the magnetic polarization is proportional to the product of the static magnetic field and the volume magnetic susceptibility, $\chi_{\mathrm{v}}$, we have to know the precise values of the $\chi_{\mathrm{V}}$ of the relevant sample solutions for the correction, but reliable values are rarely available. This problem was pointed out already 30 years ago [2].

\footnotetext{
*Lecture presented at the European Molecular Liquids Group (EMLG) Annual Meeting on the Physical Chemistry of Liquids: Novel Approaches to the Structure, Dynamics of Liquids: Experiments, Theories, and Simulation, Rhodes, Greece, 7-15 September 2002. Other presentations are published in this issue, pp. 1-261.

ॠCorresponding author
} 
For the last five years, we have been trying to develop the external double reference method for exact determination of ${ }^{1} \mathrm{H}$ - and ${ }^{13} \mathrm{C}$-chemical shifts of aqueous solutions at a wide range of concentrations or temperatures on a unified scale, by extending the bulbed capillary method invented by Momoki and Fukazawa [3]. In their method, a capillary tube with a blown-out sphere is used as a container of the reference substance, which is inserted into the sample tube. The NMR spectrum thus obtained is composed of a set of sample peaks and two reference peaks attributable to the reference substance in the capillary and in the bulb. The double reference peaks allow us to evaluate the precise $\chi_{\mathrm{V}}$ in situ necessary for the correction.

In this paper, we present the principle of the external double reference method and the exact temperature dependences of the chemical shifts for water and methanol as the first data determined on a unified scale. We also report the data of $\chi_{\mathrm{v}}$ 's for several deuterated solvents useful for the determination of the shape factor for the bulbed capillary tube. Application of the method to the concentration dependence of chemical shift on a unified scale has been reported elsewhere [4-7].

\section{PRINCIPLE OF EXTERNAL DOUBLE REFERENCE METHOD}

On application of an external field $H$ to a sample, a nuclear spin in the sample feels additionally the Lorentz field and the demagnetizing field together with the hyperfine field from the surrounding electrons. The Lorentz field is equal to the field in a microcavity in the medium in which the probe nuclear spins exist and is given by $(4 \pi / 3) \chi_{\mathrm{v}} H$ in terms of the $\chi_{\mathrm{v}}$ of the medium. This field causes a shift of the resonance field from that for free nuclear spin $H_{0}$. Hereafter, the shift will be denoted as $\left(H-H_{0}\right) / H_{0}$ in ppm, so that the shift due to the Lorentz field is ${ }^{\mathrm{L}} \delta=(4 \pi / 3) \chi_{\mathrm{v}} \times 10^{6}$.

When a reference tube in a sample tube is mounted at the center of the superconducting magnet, and parallel to the external magnetic field $H$, the reference substance in the capillary tube as well as the sample feels no demagnetizing field as long as the lengths of the tubes are much larger than their radii. Consequently, in this configuration, the shift of a resonance peak, $\delta_{\text {sum }}$, is the sum of the shifts due to the hyperfine interactions, ${ }^{\mathrm{h}} \delta$, and the Lorenz field, ${ }^{\mathrm{L}} \delta$;

$$
\delta_{\text {sum }}={ }^{\mathrm{h}} \delta+{ }^{\mathrm{L}} \delta={ }^{\mathrm{h}} \delta+(4 \pi / 3) \chi_{\mathrm{v}} \times 10^{6} .
$$

In the external reference method using a cylindrical capillary tube, an apparent chemical shift, $\delta_{\text {obs }}$, is measured as the spacing between a signal from molecules in the outer sample tube, $\delta_{\text {sam }}$, and that in the inner reference tube, $\delta_{\text {ref }}$. Equation 1 gives the following equation for $\delta_{\text {obs }}$;

$$
\delta_{\text {obs }}=\delta_{\text {sam }}-\delta_{\text {ref }}={ }^{\mathrm{h}} \delta_{\text {sam }}-{ }^{\mathrm{h}} \delta_{\text {ref }}+(4 \pi / 3)\left(\chi_{\text {sam }}-\chi_{\text {ref }}\right) \times 10^{6},
$$

where $\chi_{\text {sam }}$ and $\chi_{\text {ref }}$ the $\chi_{\mathrm{v}}$ 's of the sample and the reference substance, respectively. Since the chemical shift we need, $\delta$, is the difference in the shifts due to the hyperfine interaction between the sample, ${ }^{\text {h }} \delta_{\text {sam }}$, and the reference, ${ }^{\text {h }} \delta_{\text {ref }}$, in eq. $2, \delta$ can be obtained from $\delta_{\text {obs }}$ after the correction for the Lorentz field as

$$
\delta={ }^{\mathrm{h}} \delta_{\text {sam }}-{ }^{\mathrm{h}} \delta_{\text {ref }}=\delta_{\text {obs }}-(4 \pi / 3)\left(\chi_{\text {sam }}-\chi_{\text {ref }}\right) \times 10^{6} .
$$

To deduce the exact $\delta$ from $\delta_{\text {obs }}$, we need to know the precise value of $\left(\chi_{\text {sam }}-\chi_{\text {ref }}\right)$ for each measurement. We can obtain $\left(\chi_{\text {sam }}-\chi_{\text {ref }}\right)$ in situ by using a capillary tube with a bulb as shown in Fig. 1. 


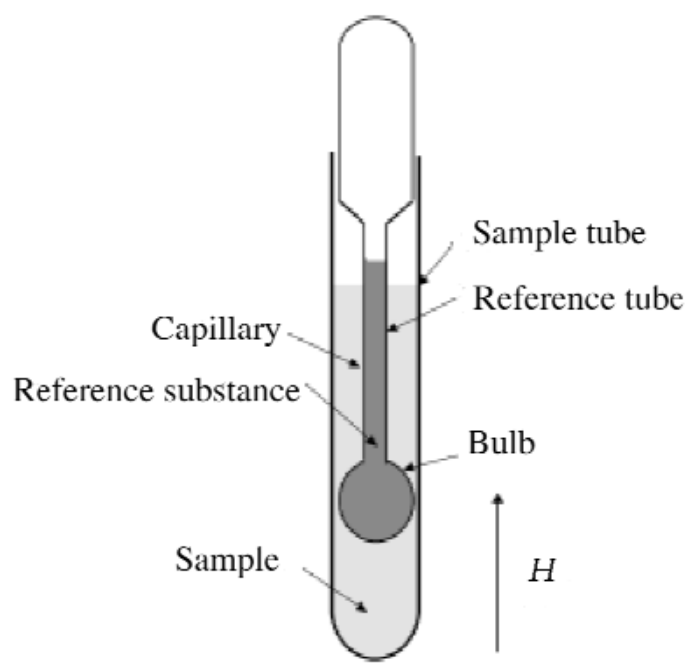

Fig. 1 A capillary tube with a bulb inserted in a sample tube.

If the bulb of the reference tube is surrounded by sample solution, the reference substance in the bulb feels a nonzero demagnetizing field equal to $\kappa\left(\chi_{\text {sam }}-\chi_{\text {ref }}\right) H$, where $-\kappa$ is the demagnetizing factor for the bulb and equal to $4 \pi / 3$ for an ideal sphere. This difference in the demagnetizing fields between the capillary and the bulb gives rise to the two reference peaks. The shift between the two peaks, $\Delta \delta_{\text {ref }}$, is

$$
\Delta \delta_{\text {ref }}=\delta_{\text {cap }}-\delta_{\text {bul }}=\kappa\left(\chi_{\text {sam }}-\chi_{\text {ref }}\right) \times 10^{6},
$$

as derived by Mulay and Harverbusch [8]. Using $\Delta \delta_{\text {ref }}$ given by $\left(\chi_{\text {sam }}-\chi_{\text {ref }}\right)$ from eq. 4 , we can obtain the exact value of the chemical shift with respect to the reference signal from the capillary tube as

$$
\delta=\delta_{\text {obs }}-(4 \pi / 3 \kappa) \Delta \delta_{\text {ref }}
$$

The $\kappa$ can be determined experimentally by measuring $\Delta \delta_{\text {ref }}$ for several samples with known $\chi_{\text {sam }}$.

The external double reference method is an ingenious technique to measure the chemical shifts of various sample solutions at different temperatures with respect to a fixed reference substance at a common single state, that is, on a unified scale. Moreover, using a vacant sample tube where $\chi_{\text {sam }}=0$, we can measure precisely the temperature dependence of the volume magnetic susceptibility for a liquid in the bulbed capillary tube. Since the mass magnetic susceptibility has insignificant temperature dependence for most of diamagnetic liquids, temperature variation of the volume susceptibility is attributable to the thermal expansion of the liquid, so that we can deduce the densities at various temperatures from the temperature dependence of the chemical shift measured by the external double reference method for vacant sample tube.

To our knowledge, no reliable data of the temperature dependence of a chemical shift has been reported except the data taken using a reference substance in a gaseous state where the reference substance has the chemical shift substantially independent of temperature $[2,9,10]$. Here we show the potential of the external double reference method for the measurement of the exact temperature dependence of chemical shift measured at a temperature, $T$, and referred to the chemical shift of a reference substance at a fixed reference temperature $T_{\mathrm{r}}$.

Experimentally, the chemical shift is always measured using the reference substance at the same temperature as the sample temperature. Figure 2 shows the schematic diagram of NMR spectra obtained by the external double resonance method at temperatures of $T$ and $T_{\mathrm{r}}$. The problem is how to deduce $\delta^{T \mathrm{r}}(T)$ from $\delta(T)$ and $\delta\left(T_{\mathrm{r}}\right)$, where the temperatures in the parentheses and the superscript refer to those 
(a): Temperature $=T$

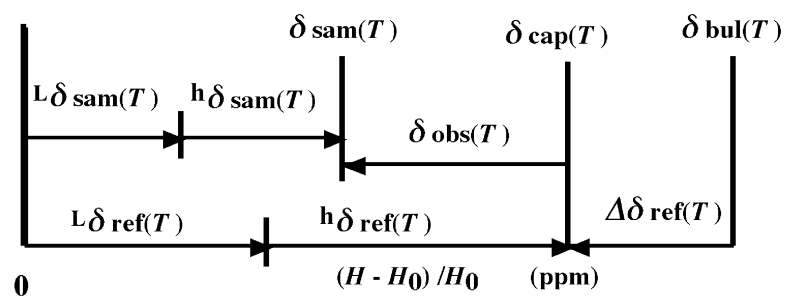

(b): Temperature $=T \mathrm{r}$

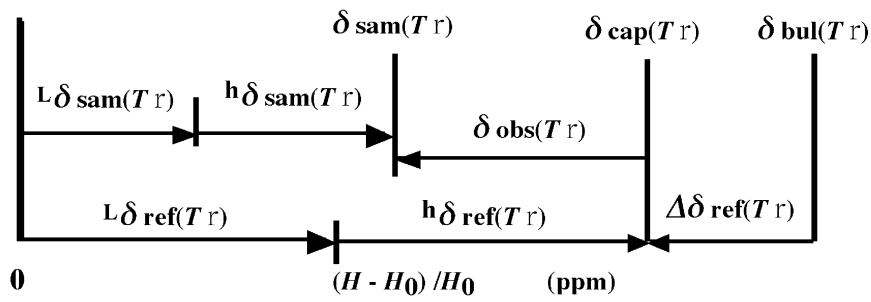

Fig. 2 Schematic diagram of NMR spectra obtained by the external double resonance method. Three thick lines stand for the resonance peaks, and $H_{0}$ is the resonance field for free nuclear spins.

of the sample solution and the reference substance solution, respectively, and $\delta(T)$ and $\delta\left(T_{\mathrm{r}}\right)$ correspond to $\delta^{T}(T)$ and $\delta^{T \mathrm{r}}\left(T_{\mathrm{r}}\right)$, respectively. From eq. 3,

$$
\delta^{T \mathrm{r}}(T)={ }^{\mathrm{h}} \delta_{\text {sam }}(T)-{ }^{\mathrm{h}} \delta_{\text {ref }}\left(T_{\mathrm{r}}\right)
$$

Figure 2 gives the shift for the signal of the reference substance in the capillary at $T$ as

$$
\delta_{\text {cap }}(T)={ }^{\mathrm{h}} \delta_{\text {sam }}(T)+{ }^{\mathrm{L}} \delta_{\text {sam }}(T)-\delta_{\text {obs }}(T) .
$$

Then, we have

$$
{ }^{\mathrm{h}} \delta_{\text {sam }}(T)=\delta_{\text {obs }}(T)-{ }^{\mathrm{L}} \delta_{\text {sam }}(T)+\delta_{\text {cap }}(T) .
$$

On the other hand, eq. 1 gives the shift at $T_{\mathrm{r}}$ as

$$
\delta_{\text {cap }}\left(T_{\mathrm{r}}\right)={ }^{\mathrm{h}} \delta_{\text {ref }}\left(T_{\mathrm{r}}\right)+{ }^{\mathrm{L}} \delta_{\text {ref }}\left(T_{\mathrm{r}}\right),
$$

which is rewritten as

$$
{ }^{\mathrm{h}} \delta_{\text {ref }}\left(T_{\mathrm{r}}\right)=\delta_{\text {cap }}\left(T_{\mathrm{r}}\right)-{ }^{\mathrm{L}} \delta_{\text {ref }}\left(T_{\mathrm{r}}\right)
$$

Substituting eqs. 7 and 8 into eq. 6 , we obtain the following equation for $\delta^{T \mathrm{r}}(T)$;

$$
\delta^{T \mathrm{r}}(T)=\delta_{\text {obs }}(T)-{ }^{\mathrm{L}} \delta_{\text {sam }}(T)+{ }^{\mathrm{L}} \delta_{\text {ref }}\left(T_{\mathrm{r}}\right)+\delta_{\text {cap }}(T)-\delta_{\text {cap }}\left(T_{\mathrm{r}}\right) .
$$

Then, using the relation ${ }^{\mathrm{L}} \delta=(4 \pi / 3) \chi_{\mathrm{v}} \times 10^{6}$, we have

$$
\begin{aligned}
\delta^{T \mathrm{r}}(T) & =\delta_{\text {obs }}(T)-(4 \pi / 3)\left\{\chi_{\text {sam }}(T)-\chi_{\text {ref }}\left(T_{\mathrm{r}}\right)\right\} \times 10^{6}+\delta_{\text {cap }}(T)-\delta_{\text {cap }}\left(T_{\mathrm{r}}\right) \\
& =\delta_{\text {obs }}(T)-(4 \pi / 3)\left\{\chi_{\text {sam }}(T)-\chi_{\text {ref }}(T)+\chi_{\text {ref }}(T)-\chi_{\text {ref }}\left(T_{\mathrm{r}}\right)\right\} \times 10^{6}+\delta_{\text {cap }}(T)-\delta_{\text {cap }}\left(T_{\mathrm{r}}\right) \\
& =\delta_{\text {obs }}(T)-(4 \pi / 3 \kappa) \Delta \delta_{\text {ref }}(T)-(4 \pi / 3)\left\{\chi_{\text {ref }}(T)-\chi_{\text {ref }}\left(T_{\mathrm{r}}\right)\right\} \times 10^{6}+\delta_{\text {cap }}(T)-\delta_{\text {cap }}\left(T_{\mathrm{r}}\right) .
\end{aligned}
$$

Since we can express $\delta(T)$ as follows from eq. 5,

$$
\delta(T)=\delta_{\text {obs }}(T)-(4 \pi / 3 \kappa) \Delta \delta_{\text {ref }}(T),
$$


we finally obtain the following relation necessary to determine the temperature dependence of chemical shift by substituting eq. 10 for eq. 9 ,

$$
\delta^{T \mathrm{r}}(T)=\delta(T)-(4 \pi / 3)\left\{\chi_{\text {ref }}(T)-\chi_{\text {ref }}\left(T_{\mathrm{r}}\right)\right\} \times 10^{6}+\delta_{\text {cap }}(T)-\delta_{\text {cap }}\left(T_{\mathrm{r}}\right) .
$$

If the temperature dependence of the $\chi_{\mathrm{v}}$ for the reference substance, $\chi_{\text {ref }}(T)$, is measured directly by the external double reference method or calculated using thermal expansion coefficient in literature, $\delta^{T \mathrm{r}}(T)$ can be evaluated on a unified scale from eq. 11 since $\left\{\delta_{\text {cap }}(T)-\delta_{\text {cap }}\left(T_{\mathrm{r}}\right)\right\}$ can be obtained from the spectra at $T$ and $T_{\mathrm{r}}$ directly.

\section{EXPERIMENTS}

\section{NMR Experiments}

${ }^{1} \mathrm{H}$ - and ${ }^{13} \mathrm{C}-\mathrm{NMR}$ spectra were measured with a JEOL EX-400 NMR spectrometer operating at $400 \mathrm{MHz}$ for ${ }^{1} \mathrm{H}$ and $100 \mathrm{MHz}$ for ${ }^{13} \mathrm{C}$ at temperatures ranging from $0.0{ }^{\circ} \mathrm{C}$ to $95.0{ }^{\circ} \mathrm{C}$ with an accuracy of $\pm 0.1^{\circ} \mathrm{C}$. The temperature was calibrated with a thermocouple inserted into the sample tube set in the probe of superconducting magnet prior to the measurements.

Chemical shifts were determined by the external double reference method with bulbed capillary tubes manufactured by Shigemi Co. The capillary was $2 \mathrm{~mm}$ in diameter with a blown-out sphere of $3 \mathrm{~mm}$ in diameter at the bottom. The bulbed capillary was filled with a reference substance liquid up to $60 \mathrm{~mm}$ high and inserted into the center of the sample tube of $5 \mathrm{~mm}$ in diameter as shown in Fig. 1.

The $\kappa$ values in eq. 4 can be determined for the bulbed capillary tubes, by measuring $\Delta \delta_{\text {ref }}$ for the standard liquid samples with known $\chi_{\mathrm{V}}$, such as water, methanol, ethanol, 2-propanol, chloroform, trichloroethylene, cyclohexane, cyclohexanone, and acetone, with respect to the reference substance, TMS. Plotting the obtained $\Delta \delta_{\text {ref }}$ as a function of the $\chi_{\mathrm{v}}$ for four standard samples, chloroform, trichloroethylene, cyclohexane, and cyclohexanone, we evaluated the $\kappa$ value for one of the TMS capillary tubes as shown by the line A in Fig. 3. The slope of the line gives $\kappa=-4.18 \pm 0.015$ close to the value for a perfect sphere, $-4 \pi / 3=-4.19$. The error comes predominantly from the inaccuracy of the reported value of the volume magnetic susceptibilities of the standard samples.

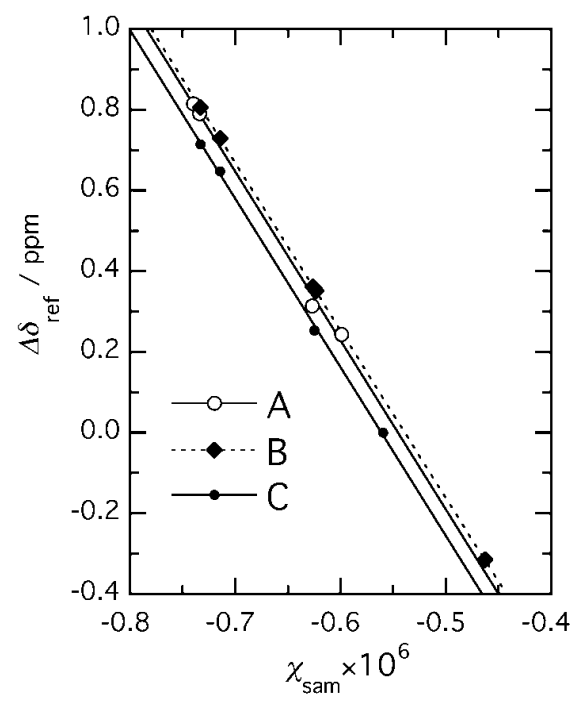

Fig. 3 Plots of $\Delta \delta_{\text {ref }}$ vs. $\chi_{\text {sam }}$ to determine the values of $\kappa$ for three tubes. (A) Trichloroethylene, chloroform, cyclohexane, and cyclohexanone were used as standard samples for a TMS reference tube. (B) and (C) Chloroform$d$, heavy water, cyclohehane- $d_{12}$, acetone- $d_{6}$, dimethylsulfoxide- $d_{6}$, and hexamethyldisiloxane were used as standard samples for TMS and HMDO reference tubes, respectively. 


\section{Chemical substances}

TMS and hexamethyldisiloxane (HMDO) from Aldrich were used as reference substances. Chloroform- $d(100$ atom $\% \mathrm{D})$, benzene- $d_{6}(100$ atom $\% \mathrm{D})$, cyclohexane- $d_{12}(99.9$ atom $\% \mathrm{D})$, and dimethylsulfoxide- $d_{6}(99.95$ atom $\% \mathrm{D}$ ) were also from Aldrich. Water was distilled twice after deionization. Methanol was spectra grade from Wako Pure Chemicals.

\section{RESULTS AND DISCUSSION}

\section{Volume magnetic susceptibilities of deuterated solvents}

NMR spectrometers are usually equipped with an automatic shimming system utilizing deuterium nuclei. But the system is not applicable to the calibration of the reference tubes using nondeuterated solvents. If deuterated solvents with known $\chi_{\mathrm{v}}$ are available, the calibration would be much easier. We measured $\Delta \delta_{\text {ref }}$ values for the deuterated solvents by the external double reference method to obtain the $\chi_{\mathrm{v}}$ values at $25.0^{\circ} \mathrm{C}$ listed in Table 1 in unit of $10^{-6}$. Here, $\chi_{\mathrm{v}}$ is a dimensionless quantity.

Table 1 Volume magnetic susceptibilities of deuterated solvents obtained by external double reference method using TMS and $\mathrm{HMDO}$ as the reference substance at $25.0^{\circ} \mathrm{C}$.

\begin{tabular}{lccc}
\hline & $\begin{array}{c}\text { TMS } \\
\left(\times 10^{-6}\right)\end{array}$ & $\begin{array}{c}\text { HMDO } \\
\left(\times 10^{-6}\right)\end{array}$ & $\begin{array}{c}\text { Average } \\
\left(\times 10^{-6}\right)\end{array}$ \\
\hline Chloroform- $d$ & -0.741 & -0.733 & -0.737 \\
Cyclohexane- $d_{12}$ & -0.634 & -0.625 & -0.630 \\
Benzene- $d_{6}$ & -0.634 & -0.603 & -0.619 \\
DMSO- $d_{6}$ & -0.637 & -0.615 & -0.626 \\
\hline
\end{tabular}

Making use of the newly determined $\chi_{\mathrm{v}}$ values for the deuterated solvents in addition to deuterated acetone and heavy water, we calibrated new bulbed tubes containing TMS or HMDO as the reference substance. The lines B and C in Fig. 3 are the calibration curves that yield the $\kappa$ values $-4.15 \pm 0.02$ and $-4.17 \pm 0.025$, respectively. They are also close to the ideal value.

\section{Temperature dependence of chemical shifts}

We carried out the measurements of ${ }^{1} \mathrm{H}$-chemical shift of water over the temperature range from 0 to $95{ }^{\circ} \mathrm{C}$ by the external double reference method using the HMDO reference tube of $\kappa=-4.17$. Equations 10 and 11 were applied to obtain $\delta_{\mathrm{H} 2 \mathrm{O}}{ }^{T \mathrm{r}}(T)$ for $T_{\mathrm{r}}=25.0{ }^{\circ} \mathrm{C}$ from $\delta_{\mathrm{obs}}(T)$ of the water proton, $\Delta \delta_{\text {ref }}(T)$, and $\left\{\delta_{\text {cap }}(T)-\delta_{\text {cap }}\left(T_{\mathrm{r}}\right)\right\}$ at each temperature $T$. The value $-(4 \pi / 3)\left\{\chi_{\text {ref }}(T)-\chi_{\text {ref }}\left(T_{\mathrm{r}}\right)\right\}$ for HMDO was evaluated to be $0.003306(25-T)$ from the data of mass magnetic susceptibility $\chi_{\mathrm{m}}$ and density $\rho$. Thus, $\delta_{\mathrm{H} 2 \mathrm{O}}^{25}(T)$ can be obtained by the following equation,

$$
\delta_{\mathrm{H} 2 \mathrm{O}}^{25}(T)=\delta_{\mathrm{H} 2 \mathrm{O}}(T)+0.003306(25-T)+\delta_{\text {cap }}(T)-\delta_{\text {cap }}\left(T_{\mathrm{r}}\right) .
$$

In Fig. 4, the values of $\delta_{\mathrm{H} 2 \mathrm{O}}{ }^{25}(T)$ and $\delta_{\mathrm{H} 2 \mathrm{O}}(T)$ are plotted against temperature. $\delta_{\mathrm{H} 2 \mathrm{O}}{ }^{25}(T)$ decreases with temperature, which is attributable to the dissociation of hydrogen bonds among $\mathrm{OH}$ groups in water. Equation 12 indicates that the difference between $\delta_{\mathrm{H} 2 \mathrm{O}}{ }^{25}(T)$ and $\delta_{\mathrm{H} 2 \mathrm{O}}(T)$ corresponds to the changes in the chemical shift and in the $\chi_{\mathrm{v}}$ of the reference substance with temperature. The observed difference was found to be less than $0.02 \mathrm{ppm}$ from Fig. 4. This result shows that HMDO is an excellent reference substance to obtain $\delta(T)$ for ${ }^{1} \mathrm{H}$ over a wide range of temperature and that we can estimate $\delta^{25}(T) \approx \delta(T)$ as long as ${ }^{1} \mathrm{H}$-chemical shift is measured over a narrow temperature range in reference to HMDO. 


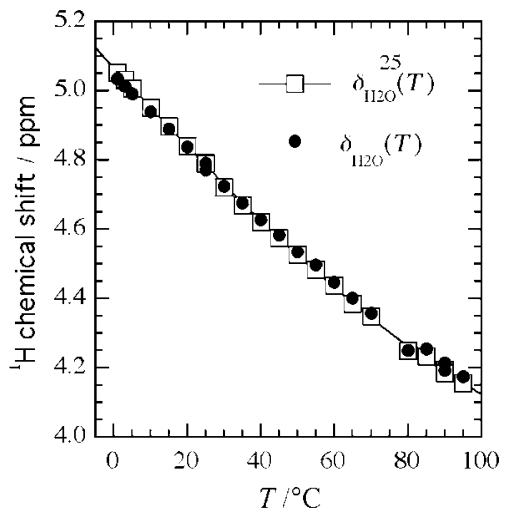

Fig. 4 Plots of $\delta_{\mathrm{H} 2 \mathrm{O}}{ }^{25}(T)$ and $\delta_{\mathrm{H} 2 \mathrm{O}}(T)$ for water ${ }^{1} \mathrm{H}$ vs. temperature.

Hindman [11] measured the chemical shifts of water by referring to the ${ }^{1} \mathrm{H}$ in methane gas sealed in a capillary reference tube, and corrected the observed chemical shift in terms of the $\chi_{\mathrm{v}}$ at every temperature calculated from $\chi_{\mathrm{m}}$ and density data. In Fig. $5, \delta_{\mathrm{H} 2 \mathrm{O}}{ }^{25}(T)$ are plotted vs. $T$ together with the data by Hindman after normalizing the value at $25.0{ }^{\circ} \mathrm{C}$. Hindman referred the chemical shift to the methane ${ }^{1} \mathrm{H}$ at every temperature. Consequently, he did not determine the chemical shift on a unified scale, but only $\delta_{\mathrm{H} 2 \mathrm{O}}(T)$ corrected with the density data. Notwithstanding, since he used a gaseous reference substance of which chemical shift shows no temperature dependence, it is hard to recognize the deviation of the data by Hindman from the $\delta_{\mathrm{H} 2 \mathrm{O}}{ }^{25}(T)$ we obtained. Thus, gaseous reference substance is confirmed to be very convenient for studying the temperature dependence of chemical shift.

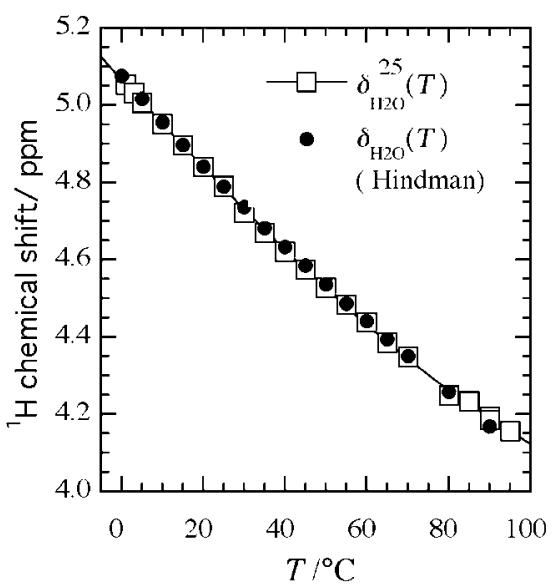

Fig. 5 Comparison of ${ }^{1} \mathrm{H}$-chemical shift of water between $\delta_{\mathrm{H} 2 \mathrm{O}}{ }^{25}(T)$ by us and the data by Hindman.

$\chi_{\mathrm{v}}$ 's of water were evaluated by measuring $\Delta \delta_{\text {ref }}$ in eq. 4 and are plotted against temperature in Fig. 6 to compare with the $\chi_{\mathrm{v}}$ values calculated from the mass susceptibility $\chi_{\mathrm{m}}$ and density $\rho$. The difference between the two susceptibilities is less than $0.006 \times 10^{-6}$ or $0.8 \%$. This excellent agreement indicates the reliability of $\chi_{\text {sam }}$ in eq. 4 obtained by the external double reference method.

Figures 7 and 8 show the temperature dependences of ${ }^{1} \mathrm{H}$-chemical shifts of $\mathrm{OH}$ and $\mathrm{CH}_{3}$, $\delta_{\mathrm{OH}}{ }^{25}(T)$ and $\delta_{\mathrm{CH} 3}{ }^{25}(T)$, and of ${ }^{13} \mathrm{C}$-chemical shift of the $\mathrm{CH}_{3}, \delta_{\mathrm{CH} 3}{ }^{25}(T)$, in methanol, respectively. The data $\delta_{\underline{\mathrm{CH}} 3}(T)$ of the ${ }^{13} \mathrm{C}$ in $\mathrm{CH}_{3}$ is also plotted in Fig. 8 . 


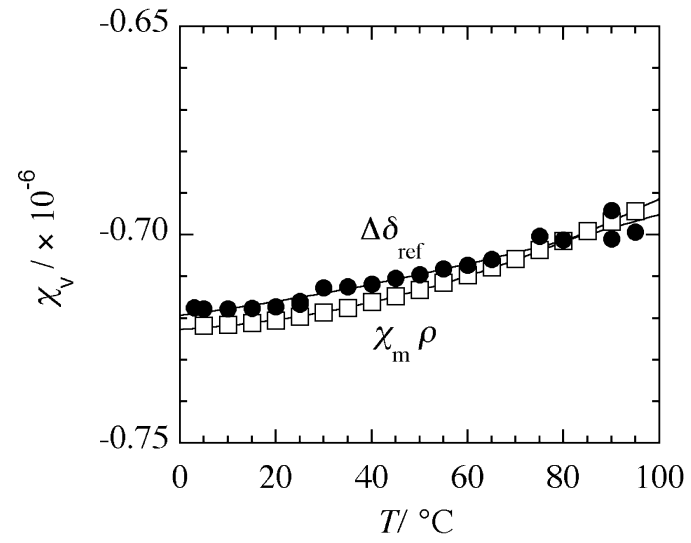

Fig. 6 Comparison of $\chi_{\mathrm{v}}$ obtained from $\Delta \delta_{\text {ref }}$ with the values calculated from $\chi_{\mathrm{m}}$ and density $\rho$.

The $\delta_{\mathrm{OH}}{ }^{25}(T)$ of methanol decreases with temperature and the decrease is also attributable to the dissociation of hydrogen bonds among $\mathrm{OH}$ groups in liquid methanol. This depolarization of $\mathrm{O}-\mathrm{H}$ bond should result in an increase in electron density around the $\mathrm{CH}_{3}$ carbon and the decrease in $\delta_{\mathrm{CH} 3}{ }^{25}(T)$. Contrary to the expectation, however, the constant $\delta_{\mathrm{CH} 3}{ }^{25}(T)$ and the increasing $\delta_{\mathrm{CH} 3}{ }^{25}(T)$ are observed. This contradiction might be interpreted in terms of the temperature dependence of dispersion force and blue-shifting $\mathrm{CH} \cdots \mathrm{O}$ hydrogen bond [7]. This is another example of interesting results of the present work.

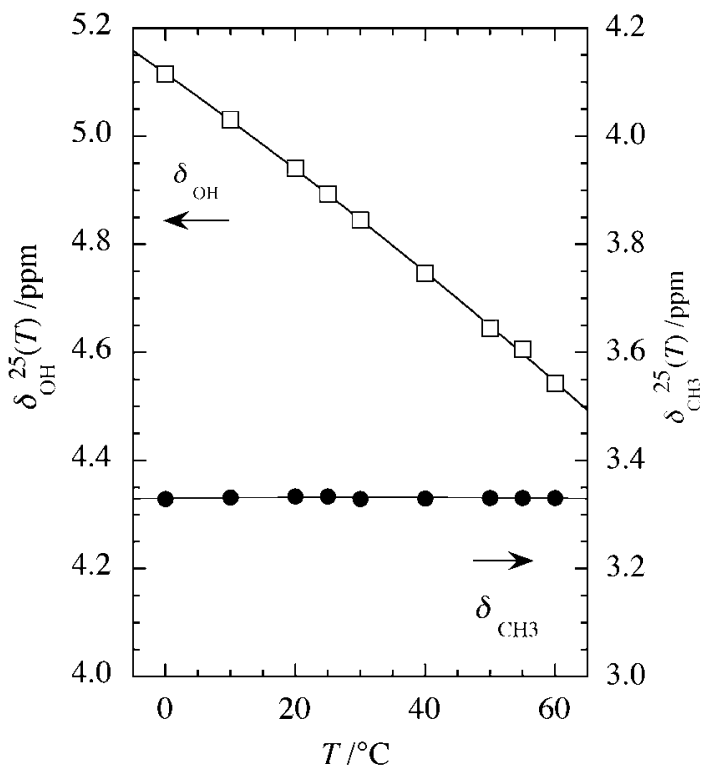

Fig. 7 Temperature dependences of $\delta_{\mathrm{OH}}{ }^{25}(T)$ and $\delta_{\mathrm{CH} 3}{ }^{25}(T)$ for ${ }^{1} \mathrm{H}$ of $\mathrm{OH}$ and $\mathrm{CH}_{3}$ in methanol.

Whereas the difference in ${ }^{1} \mathrm{H}$-chemical shift between $\delta_{\mathrm{CH} 3}{ }^{25}(T)$ and $\delta_{\mathrm{C} \underline{H} 3}(T)$ is the same as that between $\delta_{\mathrm{H} 2 \mathrm{O}}{ }^{25}(T)$ and $\delta_{\mathrm{H} 2 \mathrm{O}}(T)$, as seen from eq. 12, and is not so significant, a rather substantial difference in ${ }^{13} \mathrm{C}$-chemical shift between $\delta_{\mathrm{CH} 3}{ }^{25}(T)$ and $\delta_{\underline{\mathrm{CH}} 3}(T)$ was observed for the $\mathrm{CH}_{3}$ in methanol. The difference amounts to more than $0.2 \mathrm{ppm}$ at $60{ }^{\circ} \mathrm{C}$ as shown in Fig. 8. The difference results from the increase in the ${ }^{13} \mathrm{C}$-chemical shifts of the $\mathrm{CH}_{3}$ in the reference substance, $\mathrm{HMDO}$, the same as the 


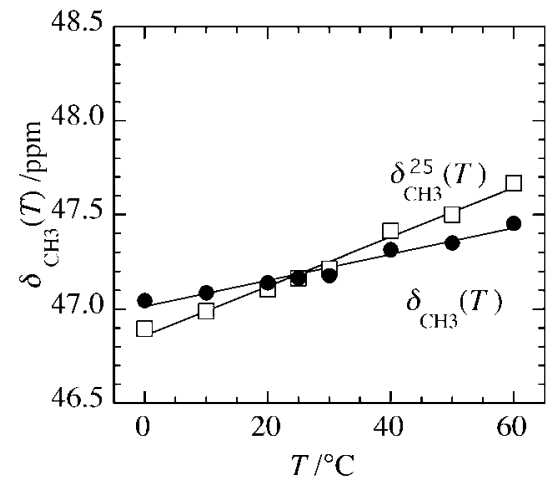

Fig. 8 Temperature dependence of $\delta_{\underline{\mathrm{CH}} 3}{ }^{25}(T)$ and $\delta_{\underline{\mathrm{CH}} 3}(T)$ for ${ }^{13} \mathrm{C}$ of $\mathrm{CH}_{3}$ in methanol.

increase in $\delta_{\mathrm{CH} 3}{ }^{25}(T)$ in methanol with temperature. These are examples of interesting results of the chemical shift measurements on a unified scale by the external double reference method.

\section{CONCLUSION}

In the present paper, the details of the external double reference method are discussed. We can determine chemical shifts of samples in a variety of conditions on a unified scale according to eq. 9; this method allows us to evaluate the exact temperature dependence of the chemical shift of a sample on a unified scale by the correction relevant to the temperature variation of the volume magnetic susceptibility and the chemical shift of the reference substance. The wide applicability of the method is demonstrated by showing some examples of the temperature dependence studies of chemical shift and the measurements of volume magnetic susceptibility in situ.

We have found that the correction terms in eq. 11 come from the changes in the $\chi_{\mathrm{V}}$ and the chemical shift of the reference substance. Our results show that $\left\{\delta^{25}(T)-\delta(T)\right\}$ is relatively small for ${ }^{1} \mathrm{H}$-chemical shift in reference to HMDO, but substantial for ${ }^{13} \mathrm{C}$-chemical shift. Although the mechanisms of the temperature variation of chemical shifts in solutions are some future problems, the external double reference method will provide reliable experimental results that will stimulate theorists.

\section{ACKNOWLEDGMENTS}

We thank Associate Prof. Shiro Maeda of Fukui University for his help and support for measuring NMR spectra. This work was supported by a Grant-in-Aid for a Scientific Research program [13640504] from the Ministry of Education, Culture, Sports, Science and Technology of Japan.

\section{REFERENCES}

1. C. J. Jameson. Ann. Rev. Phys. Chem. 47, 135 (1996).

2. P. C. Bradford, P. Cross, T. Schleich. Org. Magn. Reson. 10, 82 (1977).

3. K. Momoki and Y. Fukazawa. Anal. Chem. 62, 1665 (1990).

4. K. Mizuno, T. Ochi, Y. Shindo. J. Chem. Phys. 109, 9502 (1998).

5. K. Mizuno, S. Imafuji, T. Ohta, S. Maeda. J. Phys. Chem. 104, 11001 (2000).

6. K. Mizuno, Y. Kimura, H. Morichika, Y. Nishimura, S. Shimada, S. Maeda, S. Imafuji, T. Ochi. J. Mol. Liq. 85, 139 (2000).

7. K. Mizuno, S. Imafuji, T. Fujiwara, T. Ohta, Y. Tamiya. J. Phys. Chem. 107, 3972 (2003).

8. L. N. Mulay and M. Harverbusch. Rev. Sci. Instrum. 35, 756 (1964). 
9. A. K. Jameson and C. J. Jameson. J. Am. Chem. Soc. 95, 8559 (1973).

10. F. Morin, M. S. Solum, D. James. M. David, D. K. Dalling. J. Magn. Reson. 48, 138 (1982).

11. J. C. Hindman. J. Chem. Phys. 44, 12 (1966). 